\title{
Regional Metastases in Well-Differentiated Thyroid Carcinoma: Pattern of Spread
}

Yoav Yanir, MD; Ilana Doweck, MD

Objectives/Hypothesis: To determine the pattern of spread of WDTC to regional lymph nodes, in patients who presented with clinically positive nodes.

Study Design: Retrospective chart review.

Materials and Methods: Between October 2001 and December 2006, a total of 27 consecutive patients (12 males, 15 females) with clinical evidence of cervical metastasis of well-differentiated thyroid carcinoma (WDTC) underwent 28 neck dissections (ND) with a mean follow-up 33.7 months. Papillary carcinoma was found in 24 patients and follicular carcinoma in 3.

All neck dissection specimens were separated during surgery into levels, and analysis was done with respect to the levels of the neck.

Clinical and demographic parameters were correlated to the pathologic parameters, including number of pathologic nodes, size of tumor, and the patient's age, with univariate and multivariate analysis.

Results: The mean number of pathologic nodes in ND specimen was 6.7. The predominant site of metastasis was level VI (95\%), followed by level III (68\%), level IV (57\%), and level II (54\%). Metastases above the XI nerve were found in $7 \%$ of the patients. Level V showed $20 \%$ of nodal metastasis. A correlation was found between size of primary tumor and number of positive pathologic lymph nodes $(P=.02)$ and an inverse correlation between the age of the patient and the number of pathologic nodes $(P=.043)$.

Conclusions: The high incidence of metastatic disease in levels II through VI supports the recommendation for posterolateral and anterior ND in patients with WDTC and clinically positive nodes. The correlation between tumor size, the age of the patient, and the number of positive nodes is an interesting finding that warrants further study.

From the Department of Otolaryngology, Head and Neck Surgery (Y.Y., I.D.), Carmel Medical Center, Haifa, Israel, and the Bruce Rappaport Faculty of Medicine, Technion-Israel Institute of Technology (I.D.), Haifa, Israel.

Editor's Note: This Manuscript was accepted for publication September 11, 2007.

Presented at the 3rd World Congress of the International Federation of the Head and Neck Oncologic Societies, Prague, Czech Republic, June $29,2006$.

Send correspondence to Ilana Doweck, MD, Department of Otolaryngology, Head and Neck Surgery, Carmel Medical Center, 7 Michal Street, Haifa 34362, Israel. E-mail: idoweck@netvision.net.il

DOI: 10.1097/MLG.0b013e31815ae3e4
Key Words: Well-differentiated thyroid carcinoma, regional metastases, neck dissection.

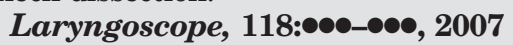

\section{INTRODUCTION}

Well-differentiated thyroid cancer (WDTC) is the most common form of thyroid gland malignancy, accounting for $70 \%$ to $80 \%$ of all cases. Many studies have shown a high percentage of metastatic lymph nodes in WDTC ranging from $30 \%$ to $80 \% .^{1-2}$

Several researchers have addressed the question of whether there is a correlation between cervical metastatic disease and survival. While previous studies failed to show any association, recent publications have shown that positive lymph nodes have a prognostic value as to recurrence of the disease and survival.

WDTC has an overall excellent prognosis with a 10year survival rate of over $90 \%$. These outstanding results have raised a question regarding the extension of surgical intervention in the neck with clinical findings of metastases. Several options have been suggested addressing positive lymph nodes in the neck, ranging from refrain from any surgical intervention, through random lymph node biopsy, i.e., "berry picking" (removal of gross metastatic nodes that could be found at the surgical bed of the thyroid), sentinel lymph node biopsy, and neck dissection. Neck dissection (ND) was proposed at a variety of extensions, including radical ND, modified ND, and various selective ND. ${ }^{3-6}$ The pattern of spread of regional metastasis from WDTC is crucial to determine the extent of neck dissection needed to be performed in patients with clinical regional metastasis. There are few studies that address this issue, but there are still insufficient data regarding the pattern of spread of cervical metastasis in WDTC. ${ }^{7-9}$

In this study, we evaluated the pattern of spread to regional lymph nodes metastasis of WDTC in patients presenting with clinically positive nodes.

\section{MATERIALS AND METHODS}

The study included all consecutive patients who underwent ND for the treatment of clinically positive metastatic lymph nodes of WDTC at the Department of Otolaryngology, Head and Neck Surgery, Carmel Medical Center, Haifa, Israel, between October 2001 to December 2006. We retrospectively reviewed the 
medical records of patients as well as pathologic reports. The indication to perform ND in all patients but one was a neck mass diagnosed on a physical examination or computed tomography (CT) scan. The remaining dissection was performed after a metastatic lymph node was found in a central compartment dissection during thyroidectomy. Diagnosis of thyroid carcinoma was done by fine-needle aspiration biopsy (FNAB) followed by intraoperative frozen section. All patients underwent preoperative CT scan, either before the diagnosis, to evaluate neck mass, or after the diagnosis of metastatic disease, to evaluate the extent of the lymphatic spread. In the latter group, there was no use of contrast to avoid iodine load prior to radioactive iodine treatment.

All patients underwent total thyroidectomy and selective neck dissection. Sixteen patients had their neck dissection done simultaneously with the thyroidectomy, whereas 11 patients had an interval neck dissection due to regional failure that was done 0.5 to 120 months after thyroidectomy (mean time 44.5 months, median 25.7 months).

Selective neck dissection was performed on all patients: 24 patients (25 necks) underwent SND including levels II-VI, whereas 3 patients had SND including levels II-IV and VI.

None of the patients had clinically positive nodes in level I, and therefore level I was not dissected in any of the patients.

All patients were operated by a single surgeon (I.D.), and all neck dissection specimens were separated during surgery into levels and sent to pathology as separate specimens. This provided an accurate description of the nodal disease in the neck according to the levels of the neck.

Following the thyroidectomy, all patients had radioactive $\mathrm{I}^{131}$ ablation. Therefore, patients who underwent interval neck dissection had radio-iodine treatment prior to neck dissection.

After surgery, patients were scheduled for a monthly follow-up visit in the first year and a 2 to 3 months follow-up visit in the second and third year. The following data were retrieved from the chart review: age, gender, time of follow-up, recurrence site (local, regional, or distant), status of the patients, and pathologic parameters, including size of the primary tumor in the thyroid gland, nodal yield (total number of nodes in neck dissection specimens), and number of pathologic nodes. Metastases to the neck were further evaluated with respect to the levels of the neck as described by Robbins et al. ${ }^{10}$

Statistical analysis was performed using JMP 4 for Windows (SAS Institute Inc., Cary, NC). Statistical analysis included descriptive statistics for age and gender of patients, size of lesions, follow-up time and the pathologic findings. A linear regression model was done between the number of nodal metastasis in the neck and tumor size as well as age of the patients. A multivariate analysis was done to include parameters that influence the number of pathologic nodes in neck dissection. For all analyses $P \leq .05$ was considered significant.

\section{RESULTS}

Between October 2001 and December 2006, a total of 27 consecutive patients with positive nodes of WDTC underwent 28 neck dissections. One patient had gross bilateral metastases to both lateral necks and required bilateral ND. The histologic type was papillary carcinoma in 24 (89\%) patients, whereas 3 patients (11\%) had follicular thyroid carcinoma. Two patients with papillary carcinoma (8\%) had tall cell variant.

The mean age at the initial diagnosis was $47 \pm 3.6$ years ( \pm SEM, range 21-85 years), and there were 12 $(45 \%)$ males and $15(55 \%)$ females. All patients but one underwent neck dissection due to clinical positive nodes that were diagnosed on physical examination or CT scan.
The remaining patient had ND due to metastatic lymph node found during thyroidectomy. The mean follow-up time after surgery was $33.7 \pm 7.2$ months. None of the patients had local or regional recurrence. All patients but two are now free of disease; the two remaining patients had distant metastasis to the lungs.

The mean diameter of the primary thyroid tumor was $2.2 \mathrm{~cm}$ (range $0.3-8$ ). The primary tumor size according to the TNM staging system for thyroid cancer of the American Joint Commission on Cancer ${ }^{11}$ was $\mathrm{T}_{1}$ in $13(48 \%)$ patients, $\mathrm{T}_{2}$ in $5(19 \%)$ patients, $\mathrm{T}_{3}$ in $2(7 \%)$ patients, and $\mathrm{T}_{4 \mathrm{a}}$ in $4(15 \%)$ patients. In three patients we were unable to determine the size of the primary tumor. There was no difference in tumor size between young patients $(<45$ years old) and older patients ( $>45$ years old).

The mean nodal yield (total number of nodes that were removed at each ND) was 33 nodes (range 10-74), and the mean number of pathologic nodes in each ND specimen was 6.7 (range $1-28$ ).

The predominant site for lymph node metastasis of WDTC was found in the central compartment (level VI), at the thyroid bed and the paratracheal area, in which $95 \%$ of the specimens were found to have metastasis (Fig. 1). The metastatic spread to the lateral neck was substantial and inverse to gravity. Most commonly involved in the lateral neck was level III, with $68 \%$ of ND specimen found to have metastasis. Level IV was positive for carcinoma in $57 \%$ of the patients, and level II had metastasis in $54 \%$ of

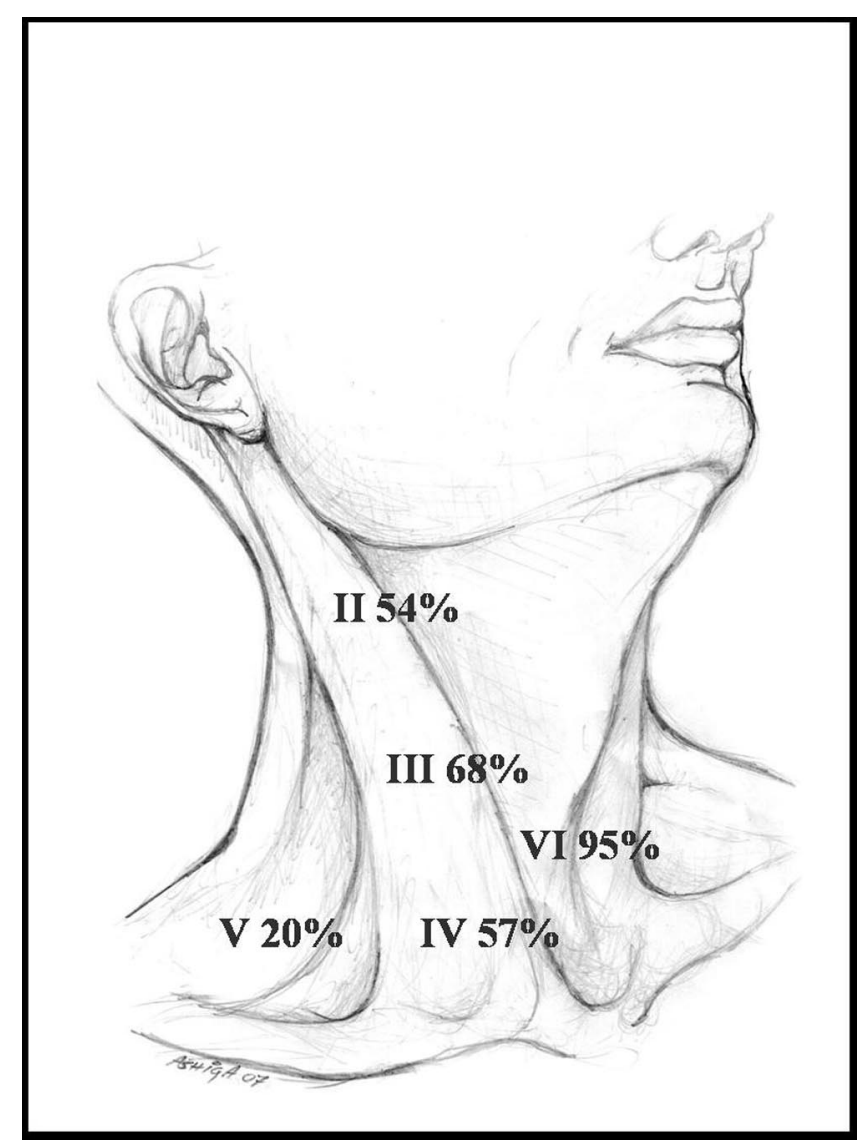

Fig. 1. Incidence of positive nodes in neck dissections by levels. 
the ND specimens. Although metastasis were predominant in level IIa (50\%), there was a small number of patients (two patients) with metastatic nodes found above the nerve, in level IIb (7\% or two patients); one of them had no metastasis in the level IIa.

In the posterior neck, at level V, $20 \%$ of the patients were found to have metastasis. Of this subgroup, four patients had metastases to level IV as well. However, two patients had metastasis to level $\mathrm{V}$ with no evidence of metastasis to level IV.

An inverse correlation was found between the age of the patient and the number of pathologic nodes $(P=$ .0425) (Fig 2). This can be expressed by the following equation:

$$
(\text { Number of positive nodes })=12.3-0.12 *(\text { age }) \text {. }
$$

A linear regression model was done to evaluate possible correlation between the number of metastatic nodes found in ND specimens and the size of the primary tumor in the thyroid gland. On this model, number of metastatic nodes in the neck is linearly correlated to tumor size in the thyroid gland ( $P=.02)$. This linear regression is demonstrated in the following equation (Fig. 3):

(Number of positive nodes) $=$

$$
2.95+1.85 *(\text { primary tumor size })
$$

A multivariate analysis was done in which the number of positive node was correlated to both primary tumor size and age of the patients. The whole model is described by the following equation:

(Number of positive nodes) $=$

$$
9.9+1.8^{*}(\text { primary tumor size })-0.14 *(\text { age }) .
$$

The whole model was significant $(P=.0038)$, with both age of the patients $(P=.01)$ and primary tumor size

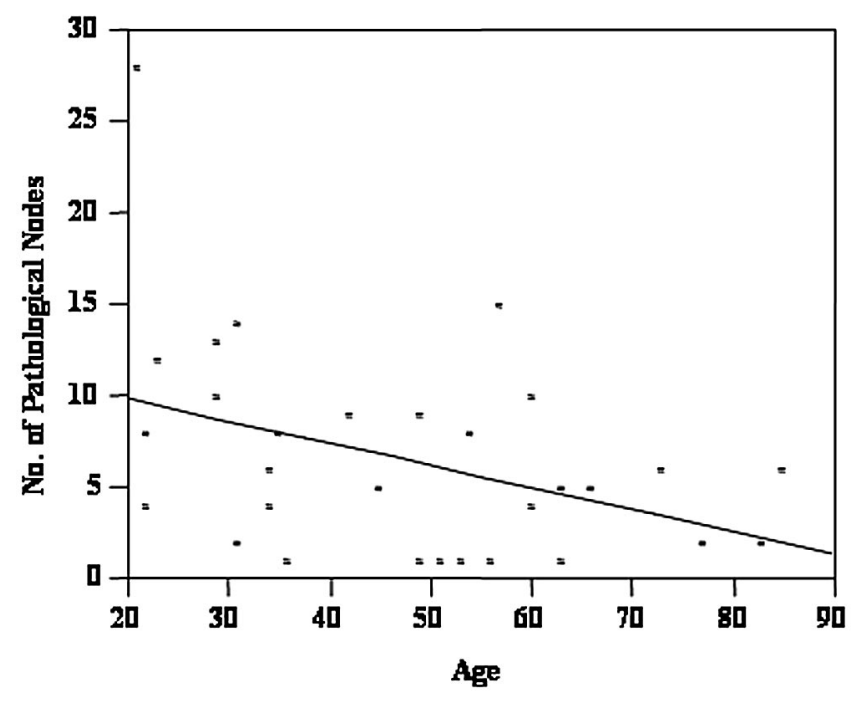

Fig. 2. Inverse correlation between patient's age and the number of metastatic nodes in the neck.

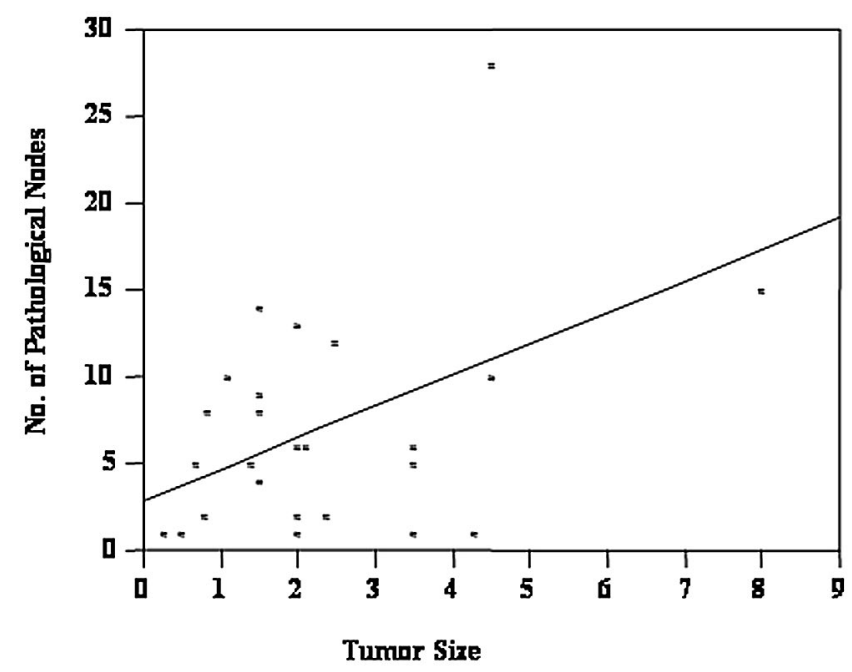

Fig. 3. Correlation between the size of the primary thyroid tumor and the number of metastatic nodes in the neck.

( $P=.0156$ ) found as independent significant predictors of number of metastatic nodes in the neck.

\section{DISCUSSION}

The management of cervical metastases in patients with WDTC is still an unresolved issue. The prognostic significance of metastases to cervical lymph nodes is still debated in the literature. Mazzaferri et al. ${ }^{1}$ demonstrated that patients with cervical node metastases had higher 30-year recurrence and mortality rates compared with those without nodal metastases. The author further showed that among older patients (over 45 years old), the presence of a cervical lymph node is even more significant as a prognostic factor for loco-regional recurrence. This finding was further confirmed by others. ${ }^{12,13}$ The debate about the clinical significance of metastatic nodes in WDTC further influences a major controversy regarding the extent of the lymphadenectomy in the neck. Several options have been suggested as to the proper way to address patients with positive cervical lymph nodes. Scheumann et al. ${ }^{3}$ compared modified neck dissection and "berry picking," and showed a significant reduction in recurrence of the disease and better overall survival for patients who were treated with modified neck dissection. Different types of neck dissection were suggested, including radical, modified, or selective neck dissection. However, the pattern of spread of regional metastasis of WDTC is crucial to determine the extent of the neck dissection.

Few studies examined the pattern of thyroid cancer metastasis. Kupferman et al. ${ }^{7}$ examined neck dissection specimens of 39 patients and showed that there was a high rate of metastases to the lateral neck, of which level III had the most prominent involvement (57\%), and the posterior neck had high involvement as well (20\%). There is no report regarding the extent of metastasis in the central compartment at that study. Pingpank et al. ${ }^{9}$ showed a similar pattern of distribution to the lateral and posterior neck; however, the rates of metastasis were 
higher: level III, 76\%; level IV, 59\%; level IIa, 43\%, and level V, $28 \%$.

Our study demonstrates that patients with clinical evidence of regional metastasis in the posterolateral neck have high rates of metastases to the central compartment (95\%). Our data also demonstrates significant involving of the lateral neck, especially to level III and IV, with $68 \%$ and $57 \%$, respectively, followed by $54 \%$ of level II.

Whereas the finding for level III is similar to previous reports, our study showed higher rates of involvement of level IV compared with previous studies.

The amount of metastasis at level II in the current study, 54\%, was found to be significant and similar to previous data. While most of the disease was found at level IIa, there were two patients (7\%) who had metastasis at level IIb, above the spinal accessory nerve. It is interesting to note that one of the patients had metastasis in level IIb with no disease at level IIa. Pingpank et al. ${ }^{9}$ reported a high rate of lymph nodes metastasis at level IIb (21\%) of whom three patients out of seven had disease above the accessory nerve with no metastasis bellow the nerve. Additionally, in 7 out of 38 patients, there was an intervening node level without disease-they called it "skip metastasis." Thus the author recommends anterior and posterolateral neck dissection for patients with lateral neck metastases of WDTC.

The amount of metastasis in the posterior neck in the current study was similar to previous reports. This supports the notion that the posterior neck should be included in the lymphadenectomy for the treatment of patients with neck involvement in WDTC.

The size of the primary thyroid tumor had been previously studied as a prognostic factor..$^{13-15}$ Reddy ${ }^{13}$ et al. showed that lymph node metastasis does not correlate with tumor size in T1 differentiated thyroid carcinoma. Shah et al. ${ }^{14}$ found that patients with tumor size between 3 to $5 \mathrm{~cm}$ have $40 \%$ greater risk of death from thyroid cancer than patients with tumor size smaller than $3 \mathrm{~cm}$. Furthermore, in patients with tumors greater than $5 \mathrm{~cm}$, the mortality risk was increased by $170 \%$ compared to patients with tumor sizes smaller than $3 \mathrm{~cm}$. Machens et al. ${ }^{15}$ found that the cumulative risk of lymph node metastasis increased linearly with increasing tumor diameter. Moreover, a correlation was found between the tumor size and the risk for distant metastasis. Our findings are in agreement with Machens et al. who suggested a correlation between primary tumor size and number of metastatic node. However, our analysis includes both the age of the patient and primary tumor size as significant and independent predictors for the extent of regional metastasis in WDTC.

\section{CONCLUSIONS}

1) Patients with WDTC and clinical positive nodes show high rates of metastases at level VI.
2) The rate of metastases is also high at the lateral neck and to a lesser extent in the posterior neck.

3) Although high in the neck, level II is frequently involved.

4) Therefore, these patients should undergo SND, including level II-VI.

5) Both tumor size and age of the patient are independent predictors for the extent of metastatic nodal disease in the neck.

\section{BIBLIOGRAPHY}

1. Mazzaferri EL, Jhiang SM. Long-term impact of initial surgical and medical therapy on papillary and follicular thyroid cancer. Am J Med 1994:97:418-428.

2. Shaha AR. Prognostic factors in papillary thyroid carcinoma and implications of large nodal metastasis. Surgery 2004; 135:237-239.

3. Scheumann GF, Gimm O, Wegener G, Hundeshagen H, Dralle H. Prognostic significance and surgical management of locoregional lymph node metastases in papillary thyroid cancer. World $J$ Surg 1994;18:559-567.

4. Caron NR, Tan YY, Ogilvie JB, et al. Selective modified radical neck dissection for papillary thyroid cancer-is level I, II and V dissection always necessary? World J Surg 2006;30:833-840.

5. Bhattacharyya N. Surgical treatment of cervical nodal metastases in patients with papillary thyroid carcinoma. Arch Otolaryngol Head Neck Surg 2003;129:1101-1104.

6. Shah MD, Hall FT, Eski SJ, Witterick IJ, Walfish PG, Freeman JL. Clinical course of thyroid carcinoma after neck dissection. Laryngoscope 2003;113:2102-2107.

7. Kupferman ME, Patterson M, Mandel SJ, LiVolsi V, Weber RS. Patterns of lateral neck metastasis in papillary thyroid carcinoma. Arch Otolaryngol Head Neck Surg 2004;130: 857-860.

8. Shaha AR, Shah JP, Loree TR. Patterns of nodal and distant metastasis based on histologic varieties in differentiated carcinoma of the thyroid. Am J Surg 1996;172:692-694.

9. Pingpank JF Jr, Sasson AR, Hanlon AL, Friedman CD, Ridge JA. Tumor above the spinal accessory nerve in papillary thyroid cancer that involves lateral neck nodes: a common occurrence. Arch Otolaryngol Head Neck Surg 2002;128: $1275-1278$.

10. Robbins KT, Clayman G, Lavine PA, et al. Neck dissection classification update: revision proposed by the American Academy of Otolaryngology-Head Neck Surgery. Arch Otolaryngol Head Neck Surg 2002;128:751-758.

11. Greene FL, Page DL, Fleming ID, et al, eds. AJCC Cancer Staging Manual, 6th edition. New York: Springer-Verlag; 2002.

12. Highes CJ, Shaha AR, Shaha JP, Loree TR. Impact of lymph node metastases in differentiated carcinoma of the thyroid: a matched-pair analysis. Head Neck 1996;18:127-132.

13. Reddy RM, Grigsby PW, Moley JF, Hall BL. Lymph node metastases in differentiated thyroid cancer under $2 \mathrm{~cm}$. Surgery 2006;140:1050-1054, discussion 1054-1055.

14. Shah JP, Loree TR, Dharker D, Strong EW, Begg C, Vlamis V. Prognostic factors in differentiated carcinoma of the thyroid gland. Am J Surg 1992;164:62-66.

15. Machens A, Holzhausen HJ, Dralle H. The prognostic value of primary tumor size in papillary and follicular thyroid carcinoma. Cancer 2005;103:2269-2273. 